\title{
Watersheds and mini-watersheds
}

\author{
Chui Ming Gemmy Cheung $\mathbb{B}^{1,2} \cdot$ Richard F. Spaide $^{3}$
}

Received: 6 February 2021 / Revised: 10 February 2021 / Accepted: 16 February 2021 / Published online: 2 March 2021

(c) The Author(s), under exclusive licence to The Royal College of Ophthalmologists 2021, corrected publication 2021

The blood flow into the choroid is delivered by the ophthalmic artery, which in $48 \%$ of people splits into, among other branches, the medial and lateral posterior ciliary arteries [1]. These, in turn, split into many short posterior ciliary arteries that pierce the sclera to supply regions of the choroid. Because the choroid has a segmental blood distribution network there is no overlap between the regions supplied by the medial and lateral posterior ciliary arteries. The area in between these regions receives blood from the terminal distribution networks of each and is called the choroidal watershed zone (CWZ). During fluorescein angiography the CWZ in such eyes can be seen as a vertical stripe of delayed filling of the choroid in the region of the optic nerve. In $39 \%$ of people there are 3 posterior ciliary arteries supplying 3 corresponding regions of the choroid. The interfaces between these regions have the potential to form a triradiate choroidal filling delay. In a relative sense, the watershed zone is thought to have decreased perfusion as compared with the adjacent areas showing normal filling.

In 1985 Hiyashi and de Laey found an association between choroidal neovascularization and CWZs, including a patient with a Fuch's spot [2]. In 2010, Wakabayashi and Ikuno showed a highly significant statistical association between myopic choroidal neovascularization ( $\mathrm{mCNV}$ ) and choroidal filling delays [3]. They ascribed the filling delays to choroidal watershed filling defects, according to the Hayreh's dictims. They also showed a significant relationship between mCNV and choroidal thinning. Recently, Sun and colleagues expanded the association beyond $\mathrm{mCNV}$ to include an association between myopic atrophy and the

Chui Ming Gemmy Cheung

gemmy.cheung.c.m@snec.com.sg

1 Singapore Eye Research Institute, Singapore National Eye Centre, Singapore, Singapore

2 Ophthalmology \& Visual Sciences Academic Clinical Program (Eye ACP), Duke-NUS Medical School, Singapore, Singapore

3 Vitreous, Macula, Retina Consultants, New York, NY, USA
CWZ [4]. In addition, they classified the filling defects into five groups, including the classic vertical through the nerve head and vertical in the parafoveal region, vertical with an extension into the macula, horizontal through the fovea, and commonly, a stellate pattern. As the stellate pattern cannot be explained by strict application of Hayreh's theories, so there must be additional factors in eyes with high myopia.

The formation of the posterior ciliary vessels occurs in early embryology producing a fixed number of vessels, usually 10-20. The ocular expansion and staphyloma formation in myopia occurs much later, and the area requiring perfusion coverage becomes much greater. The number of short posterior ciliary arteries is fixed, and therefore the area supplied by each increase and the intervening zones in between broaden. This raises the possibility that in high myopes there may be another reason for watershed zones to manifest on a unit of scale smaller than Hayreh's watershed zones. These smaller zones, between regions supplied individual short posterior ciliary arteries, form according to the same logic of a segmented choroidal distribution network. Partial confluence of these mini watershed zones may give rise to complicated patterns such as the stellate pattern. Ocular expansion in myopia, particularly in staphylomas, seems to apportion the same amount of choroidal resources to a larger area. This process has been hypothesized as the underlying cause of choroidal thinning in myopia [5]. The choriocapillaris is spread out in experimental models of myopia; Sun and colleagues show the regional blood flow through these vessels may be decreased as well. The cumulative effect may lead to decreased tissue perfusion, with atrophy and neovascularization as unfortunate consequences.

New research findings and ideas beget even more new ideas. Delineation of the relationships among age, axial length, staphyloma, and CWZ, mini or otherwise is needed. A uniform, accepted definition for CWZ, when and how long in the initial phases of the angiogram, fluorescein or indocyanine green, and how large are all factors that should be standardized. It is likely that a classification system for age-related macular degeneration may not be applicable for highly myopic eyes. One opportunity for highly myopic 
eyes is that the perforating vessels can be visualized by optical coherence tomography, and these may help in the correlation between possible short posterior ciliary arteries and the related perfusion and watershed zones.

Funding Biomedical Research Council Singapore grant: SPF2014/ 002, National Medical Research Council Open Fund Large Collaborative Grant: NMRC/LCG/004/2018.

\section{Compliance with ethical standards}

Conflict of interest The authors declare no competing interests.

Publisher's note Springer Nature remains neutral with regard to jurisdictional claims in published maps and institutional affiliations.

\section{References}

1. Hayreh SS. In vivo choroidal circulation and its watershed zones. Eye. 1990;4:273-89.

2. Hayashi K, de Laey JJ. Indocyanine green angiography of choroidal neovascular membranes. Ophthalmologica. 1985;190:30-9.

3. Wakabayashi T, Ikuno Y. Choroidal filling delay in choroidal neovascularisation due to pathological myopia. Br J Ophthalmol. 2010;94:611-5.

4. Sun J, Wang Y, Wang J. Choroidal arterial watershed zone topography and its relationship with maculopathy in highly myopic eyes. Eye (Lond) 2021. https://doi.org/10.1038/s41433-021-01427y. Online ahead of print.

5. Fujiwara T, Imamura Y, Margolis R, Slaker J, Spaide RF. Enhanced depth imaging optical coherence tomography of the choroid in highly myopic eyes. Am J Ophthalmol. 2009;148: 445-50. 